\title{
Optimization of overhaul of EMU Based on Planning Model
}

\author{
Yu Cao ${ }^{1 *}$, Hairui Zhang ${ }^{1}$, Zhong Zheng ${ }^{2}$ \\ ${ }^{1}$ College of Science, China Three Gorges University, Yichang, 443002, China. \\ ${ }^{2}$ College of Electrical Engineering \& New Energy, China Three Gorges University, Yichang 443002, \\ China.
}

\begin{abstract}
With the speed increase of the railway and the construction of the passenger dedi-cated line, the application efficiency transport of the passenger dedicated line, are directly related to the safe opera-tion of the passenger dedicated line. Therefore, China.

it is very necessary to carry out high-efficiency and highquality maintenance work for EMU. In this paper, the mainte-

maintenance process of EMU under different conditions, a mixed nonlinear programming model is established, and the software is used to solve the shortest total time of maintenance of all EMU is $541 \mathrm{~min}$. The model provides a reference for the optimization of maintenance of EMU in actual production and life, and is helpful to improve the maintenance efficiency of EMU.
\end{abstract} and maintenance quality of the EMU, as the main means of College of Science, China Three Gnance problem of EMU is studied. According to the different How to cite this article:

Keywords: Overhaul of EMU; Mixed nonlinear programming model; Genetic algorithm
${ }^{*}$ Correspondence to Author:

Yu Cao

orges University, Yichang, 443002,

Yu Cao, Hairui Zhang, Zhong Zheng. Optimization of over haul of EMU Based on Planning Model. American Journal of Basic and Applied Sciences, 2020; $3: 20$

\section{eScîPub} eSciPub LLC, Houston, TX USA. Website: http://escipub.com/ 


\section{Introduction}

According to China's Medium-and long-term Railway Network Planning (2016 Adjustment), During the period from 2016 to 2025 (long-term to 2030), we plan to build a well-developed highspeed railway network with eight vertical lines and eight horizontal lines as the skeleton, regional connection lines and inter-city railway supplement. With the gradual improvement of China's high-speed railway network and the increasing range of radiation, the demand for passenger transport will continue to be stimulated, and the operation and retention of EMU is bound to increase, which puts forward higher requirements for the transportation and inspection capacity of bullet train sections and bullet train operation stations. Therefore, the research on the maintenance optimization of EMU is of great practical significance. ${ }^{[1]}$

The motor train unit inspection and repair maintenance base mainly includes: complete maintenance depot, electrical test device, wheelset tread diagnosis device, non-falling wheel rotation equipment, wheelset and bogie replacement center, axle flaw detection device, internal cleaning and finishing equipment of EMU, external cleaning equipment of EMU, all maintenance work is carried out in the building in order not to be affected by climatic conditions and night work. In addition to the maintenance base, it is also necessary to set up a maintenance factory to carry out comprehensive inspection and maintenance of the EMU.

More than 50 bullet train stations have been built across the country. The EMU operation station is a place for maintenance and maintenance of motor cars, which is divided into different maintenance levels according to driving conditions, and different levels correspond to different processes (1) The basic procedure of maintenance: the first inspection and repair of EMU includes three processes: $a, b, c$. The EMU overhauls the motor cars in the order of $a \rightarrow b \rightarrow c$.

Tab.1 Basic data of inspection and repair of $\mathrm{CRH} 2$ bullet train

\begin{tabular}{c|c|c|c}
\hline \hline Process category & $\mathrm{a}$ & $\mathrm{b}$ & $\mathrm{c}$ \\
\hline Number of workshops (unit) & 3 & 8 & 5 \\
\hline Time spent (hours) & 1 & 2 & 1.5 \\
\hline \hline
\end{tabular}

(2) It is known that each process of different the following table: types of motor cars takes time data, as shown in

Tab.2 Time data table for each process of each type of bullet train

\begin{tabular}{|c|c|c|c|}
\hline \hline & $\mathrm{a}$ & $\mathrm{b}$ & $\mathrm{c}$ \\
\hline $\mathrm{CRH} 2$ & 1 & 2 & 1.5 \\
\hline $\mathrm{CRH} 3$ & 0.8 & 2.4 & 0.5 \\
\hline $\mathrm{CRH} 5$ & 1.3 & 2.5 & 1.5 \\
\hline $\mathrm{CRH} 6$ & 1 & 2.7 & 0.3 \\
\hline \hline
\end{tabular}

\section{Problems to be solved}

The corresponding processes of different types of EMU take different time. Given the arrival time of various types of bullet trains, it is required to calculate the total time to complete the maintenance of these trains. 


\section{Model assumptions}

Assumption 1: It is assumed that the workload of each maintenance personnel is evenly distributed.

Assumption 2: It is assumed that the maintenance machine will not break down suddenly during the maintenance process.

Assumption 3: It is assumed that all the maintenance workers can work normally, that is, there will be no accidents in the course of maintenance.

\section{Problem Analysis}

Considering that each process of different types of EMU takes different time, under the principle of "first come, first served", the total overhaul time and average waiting time may not be minimized, and the total time required to repair these cars is required to be minimum, that is, the type of motor car that takes the longest time to finish maintenance is required to be minimized. In this paper, whether the EMU enters a workshop of each process and whether it occupies the workshop at a certain time and the time when the EMU starts each process are taken as decision variables. Taking the priority relation of each process, the uniqueness of inspection and repair workshop in each process, the number of maintenance workshop in each process less than the number of available rooms, queuing relation, the starting time of the first process, the time of leaving the system and the maintenance process as constraints, and minimizing the maximum completion time as the objective function, a hybrid nonlinear programming model is established comprehensively. ${ }^{[2]}$

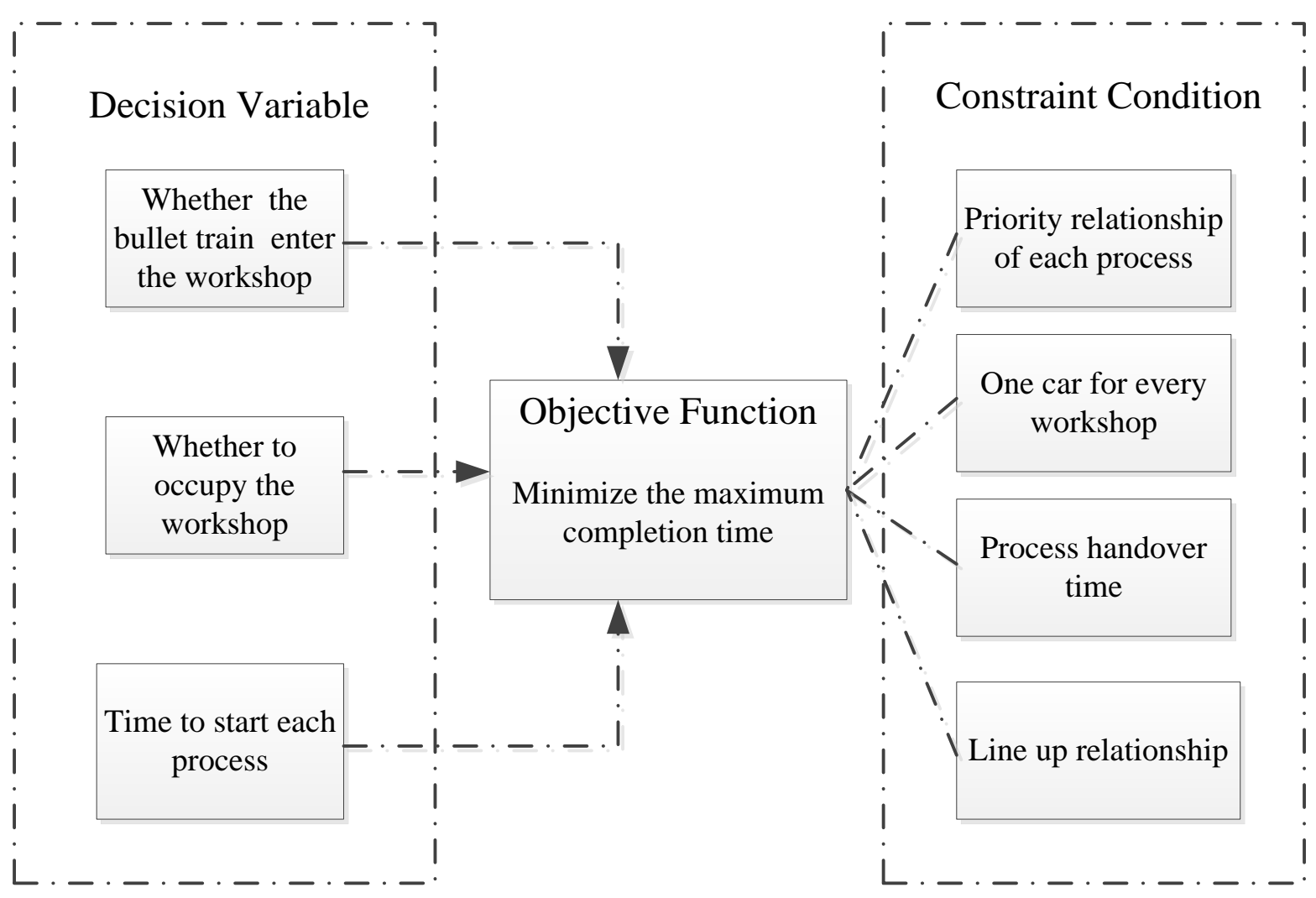

Fig.1: Idea Flowchart

\section{Analysis of data}

\subsection{Analysis of basic data of maintenance}

As the number of workshops and the time spent on bullet train maintenance will affect the main- tenance time, the basic data chart of maintenance is drawn according to the basic data table of $\mathrm{CRH} 2 \mathrm{EMU}$ given in the question, as shown below: 


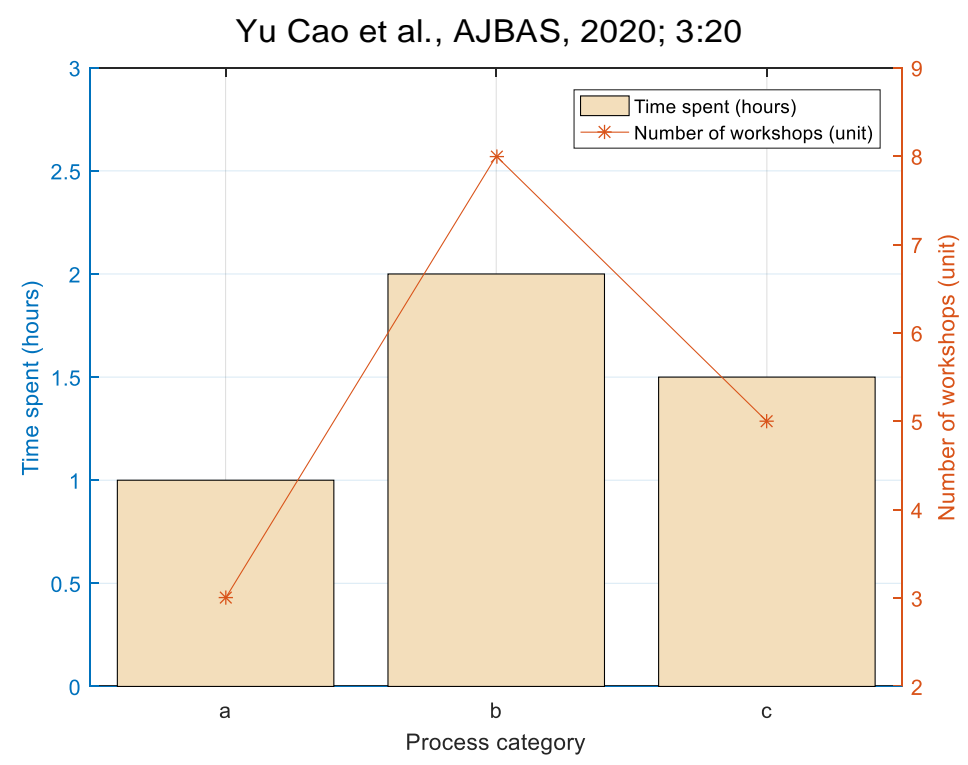

Fig.2 CRH2 EMU maintenance basic data chart

According to the observation chart, it is found that the time consuming of process $b$ is the longest and the number of workshops is the largest, and that of process $a$ is the shortest and the fewest. In order to observe the corresponding num- ber of workshops allocated to the time consumed by each process, a table of the number of workshops corresponding to the time consumed by each process is listed as follows:

Tab.3 The number of workshops corresponding to the time-consuming process

\begin{tabular}{|c|c|c|c|}
\hline \hline Process category & $\mathrm{a}$ & $\mathrm{b}$ & $\mathrm{c}$ \\
\hline Number of workshops (unit) & 3 & 2 & 5 \\
\hline Time spent (hours) & 1 & 4 & 3.3 \\
\hline Number of workshops per unit time & 3 & 4 \\
\hline
\end{tabular}

The observation table shows that the number of workshops in each process is proportional to the time spent, that is, the longer the time is, the more workshops are allocated to the process. At the same time, it can be seen from the table that the number of workshops is proportional to the number of workshops per unit time.

To observe the number of workshops per unit time, the number of units corresponding to process $a$ is the least, and process a is the first process, so there may be a situation in which there is no spare workshop in process a and cars are waiting in the arrangement. on the other hand, there are more workshops corresponding to process $b$, so it is possible that the workshop of process $b$ is not fully used. While the number of workshops in process $\mathrm{c}$ is in the middle, there may be a spare number of workshops or a motor car waiting at process $b$.

\subsection{Analysis of basic process data}

As the basic data table of the process is given in the questions of different motor cars, the working hours of each process of the four motor cars are drawn as follows: 

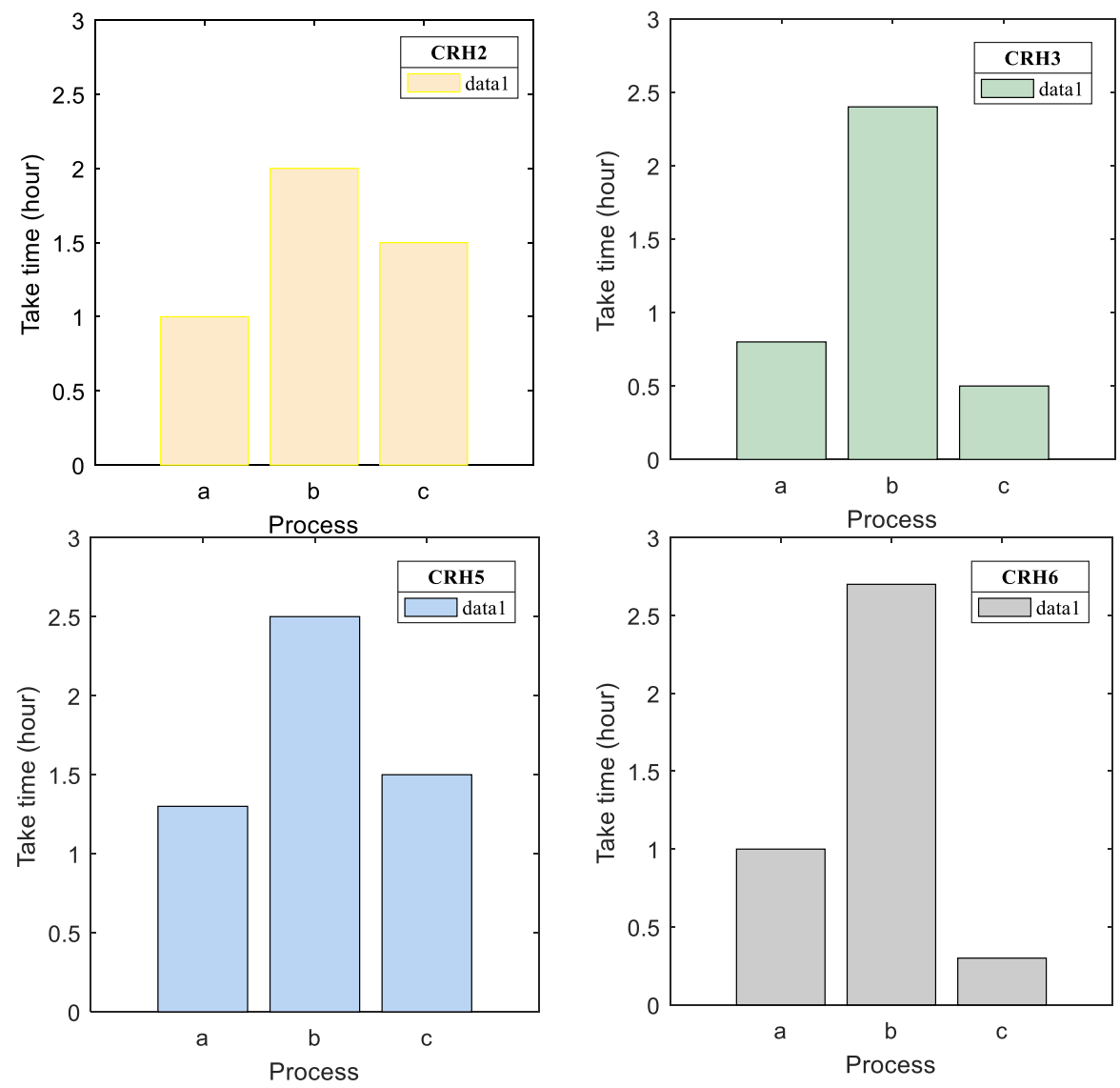

Fig. 3 Working procedure time diagram of each bullet train

According to the above picture, the time distribution of each process of the four bullet trains is similar. The working hours of $\mathrm{CHR} 2$ and $\mathrm{CHR} 5$ are roughly the same, but the duration of process $a$ is similar to that of process $\mathrm{c}$, and that of process a is slightly less than that of process $\mathrm{c}$, while that of bullet train $\mathrm{CHR} 3$ and $\mathrm{CHR} 6$ is roughly the same, with the least time of process $c$ and the longest time of process $b$.

Since the factors determining the maintenance of motor cars are the duration of the process and the number of workshops corresponding to the process, the number of workshops for each process is listed as follows:

Tab.4 Table of the number of workshops in each process

\begin{tabular}{c|c|c|c}
\hline \hline Working procedure & $\mathrm{a}$ & $\mathrm{b}$ & $\mathrm{c}$ \\
\hline Number of workshops & 3 & 8 & 5 \\
\hline \hline
\end{tabular}

From the above table, it can be found that the number of workshops corresponding to process $\mathrm{b}$ is the largest, and the number of workshops corresponding to process $a$ is the fewest. Based on the analysis of the number of workshops in each process and the time length diagram of each train process, it is found that the process $b$ of different types of motor cars takes the longest time, but the number of workshops is the most, with 8 , while process $\mathrm{c}$ takes a relatively long time, and there are 5 workshops. From this, it can be inferred that when the traffic flow is large, many cars will be stuck in the workshop of process $b$ and process $c$.

\section{Establishment and solution of the model}

\subsection{Establishment of the model}


As the time required for each process of different linear programming model is established in this types of EMU is different, it may occur that the bullet train will be repaired later to first, that is, the time-consuming type of EMU will be repaired first, so that all EMU will take the least time after overhauling. Therefore, the following hybrid non-

paper. [3]

\section{(1) Establish the objective function}

In this paper, we consider the maximum completion time minimum as the objective function, so we establish the following objective function:

$$
\min T=T_{\max }
$$

Where $T^{f}$ represents the maintenance completion time of the $f$ bullet train, and the maximum completion time of all motor cars is the maximum

$$
T_{\max }=\max \left\{T^{1}, T^{2}, \cdots, T^{11}\right\}
$$

\section{(2) Establishing constraints}

(1) The priority relationship between the processes of the same motor car, that is, in the maintenance process of the same motor car, the

$$
I_{b}^{f} \geq O_{a}^{f} ; \quad I_{c}^{f} \geq O_{b}^{f}
$$

Where $I_{a}^{f}, I_{b}^{f}$ and $I_{c}^{f}$ represent the time when the $f$ bullet train enters the process $\mathrm{a}, \mathrm{b}$ and $\mathrm{c}$ respectively, and $O_{a}^{f}, O_{b}^{f}$ and $O_{c}^{f}$ represent the time when the $f$ bullet train completes the process $a, b$ and $c$ respectively. completion time of a process should be equal to the time of entering process $b$, and the completion time of process $b$ should be equal to the time of entering process $\mathrm{c}$, so the following constraints are met:

(2) Ensure that the motor car can only be overhauled in one workshop in any process, that is, any motor car can only enter the only workshop in the same process for maintenance, so the following constraints are met:

$$
\sum_{i=1}^{3} X_{a_{i}}^{f}=1 ; \quad \sum_{j=1}^{8} X_{b_{j}}^{f}=1 ; \quad \sum_{k=1}^{5} X_{c_{k}}^{f}=1
$$

Among them, $X_{a_{i}}^{f}, X_{b_{j}}^{f}$ and $X_{c_{k}}^{f}$ respectively in- maintenance.

dicate whether the $f$ bullet train enters the $i, j$ and $k$ workshops of $\mathrm{a}, \mathrm{b}$ and $\mathrm{c}$ processes for

(3) Each process should not be interrupted during maintenance.

$$
O_{a}^{f}=I_{a}^{f}+P_{a_{i}}^{f}-1 ; O_{b}^{f}=I_{b}^{f}+P_{b_{j}}^{f}-1 ; O_{c}^{f}=I_{c}^{f}+P_{c_{k}}^{f}-1
$$

Where $I_{a}^{f}, I_{b}^{f}$ and $I_{c}^{f}$ represent the time when the $f$ bullet train enters the process $\mathrm{a}, \mathrm{b}$ and $\mathrm{c}$ respectively, and $O_{a}^{f}, O_{b}^{f}$ and $O_{c}^{f}$ represent the time when the $f$ bullet train completes the process a, b and c respectively. $P_{a_{i}}^{f}, \quad P_{b_{j}}^{f}$ and $P_{c_{k}}^{f}$

$$
P_{a_{i}}^{f}+P_{b_{j}}^{f}+P_{c_{k}}^{f}=T^{f}
$$

Where $T^{f}$ represents the completion time of the $f$ bullet train maintenance. respectively represent the maintenance time of the $f$ bullet train in the $i, j$ and $k$ workshops of the $a, b$ and $c$ processes, and the sum of the maintenance time of each process is equal to the completion time of the motor car maintenance: 
time in any stage shall not exceed the number of workshops available at that stage.

$$
\sum_{T} Y_{a_{i}}^{f}(T) \leq 3 ; \sum_{T} Y_{b_{j}}^{f}(T) \leq 8 ; \sum_{T} Y_{c_{k}}^{f}(T) \leq 5
$$

Where $Y_{a_{i}}^{f}(T) 、 Y_{b_{j}}^{f}(T) 、 Y_{c_{k}}^{f}(T)$ indicate whether the $f$ bullet train occupies the $i 、 j 、 k$ workshop of a, b, c process at the moment, $Y_{a_{i}}^{f}(T)=1$ means that the $f$ bullet train occupies the $i$ workshop of a process at the $T$ moment, $Y_{a_{i}}^{f}(T)=0$ means that the $f$ bullet train does not

occupy the $i$ workshop of a process at the $T$ moment. ( $b$ and $c$ processes are the same)

(5) The inspection and repair of process a can be carried out only after the bullet train arrives at the operation station, so the following constraints are met:

$$
t^{f} \leq I_{a}^{f}, f=1,2, \cdots, 11
$$

Where $t^{f}$ represents the time when the $f$ bullet train arrives at process a, and $I_{a}^{f}$ represents the time when the $f$ bullet train enters process a.
(6) After completing the inspection and repair of process c, the motor car can leave the motor car operation station, so the following constraints are met:

$$
\sum_{T} Y_{c_{k}}^{f}(T)=P_{c_{k}}^{f}, f=1,2, \cdots, 11
$$

Among them, $Y_{c_{k}}^{f}(T)$ indicates whether the $f$ maintenance time of the $f$ bullet train in the $k$ bullet train occupies the $k$ workshop of the $\mathrm{c}$ process at the $T$ moment, and $P_{c_{k}}^{f}$ represents the workshop of the c process.

To sum up, a mixed nonlinear programming model for the problem is obtained.

$$
\begin{gathered}
\min T=T_{\max } \\
\text { s.t }\left\{\begin{array}{l}
O_{a}^{f}=I_{a}^{f}+P_{a_{i}}^{f}-1 ; O_{b}^{f}=I_{b}^{f}+P_{b_{j}}^{f}-1 ; O_{c}^{f}=I_{c}^{f}+P_{c_{k}}^{f}-1 \\
\sum_{i=1}^{3} X_{a_{i}}^{f}=1 ; \sum_{j=1}^{8} X_{b_{j}}^{f}=1 ; \sum_{k=1}^{5} X_{c_{k}}^{f}=1 \\
P_{a_{i}}^{f}+P_{b_{j}}^{f}+P_{c_{k}}^{f}=T^{f} \\
\sum_{T} Y_{a_{i}}^{f}(T) \leq 3 ; \sum_{T} Y_{b_{j}}^{f}(T) \leq 8 ; \sum_{T} Y_{c_{k}}^{f}(T) \leq 5 \\
I_{b}^{f} \geq O_{a}^{f} ; I_{c}^{f} \geq O_{b}^{f} \\
t^{f} \leq I_{a}^{f}, f=1,2, \cdots, 11 \\
\sum_{T} Y_{c_{k}}^{f}(T)=P_{c_{k}}^{f}, f=1,2, \cdots, 11
\end{array}\right.
\end{gathered}
$$

\subsection{Solution of the model}

In this paper, we consider the time as a time axis, that is, it increases gradually from the zero time, and thinks that 00:00 is the zero time, the serial number of the bullet train coming in at $00: 16$ is 1 , and the time of the bullet train is 16 minutes, and so on. ${ }^{[4]}$
According to the established mixed nonlinear programming model, the data are solved by using MATLAB software (see appendix for the source program). The time of each bullet train going in and out of process $a, b$ and $c$ is shown in the table below. 
Yu Cao et al., AJBAS, 2020; 3:20

Tab.5 Timetable for each bullet train in and out of working procedures $a, b$ and $c$

\begin{tabular}{c|c|c|c|c|c|c|c}
\hline \hline $\begin{array}{c}\text { Train se- } \\
\text { rial num- } \\
\text { ber }\end{array}$ & $\begin{array}{c}\text { Arrival } \\
\text { time }\end{array}$ & $\begin{array}{c}\text { Enter thetime of pro- } \\
\text { cessa }\end{array}$ & $\begin{array}{c}\text { Timetoleave pro- } \\
\text { cessa }\end{array}$ & $\begin{array}{c}\text { Enter the time of pro- Timetoleave pro- } \\
\text { cessb }\end{array}$ & $\begin{array}{c}\text { Enter the time of } \\
\text { cessb }\end{array}$ & $\begin{array}{c}\text { Timetoleave pro- } \\
\text { prossc }\end{array}$ \\
\hline 1 & 16 & 16 & 76 & 76 & 196 & 196 & 286 \\
\hline 2 & 47 & 47 & 125 & 125 & 275 & 275 & 365 \\
\hline 3 & 82 & 82 & 142 & 142 & 262 & 262 & 352 \\
\hline 4 & 120 & 120 & 180 & 180 & 342 & 342 & 360 \\
\hline 5 & 141 & 141 & 189 & 189 & 333 & 333 & 363 \\
\hline 6 & 182 & 182 & 242 & 242 & 404 & 404 & 409 \\
\hline 7 & 211 & 211 & 271 & 271 & 391 & 391 & 494 \\
\hline 8 & 239 & 239 & 317 & 317 & 467 & 467 & 466 \\
\hline 9 & 241 & 242 & 290 & 290 & 434 & 434 & 493 \\
\hline 10 & 267 & 271 & 319 & 319 & 463 & 463 & 557 \\
\hline 11 & 309 & 309 & 369 & 369 & 531 & 531 & 549 \\
\hline \hline
\end{tabular}

It can be seen from the table that the time when each motor car leaves the c process, and the earliest to complete the whole maintenance process is the serial number 1 motor car, and the total maintenance time is the serial number 8 motor car, and the total maintenance time is 8 , so the total time to complete the maintenance of these motor cars is $541 \mathrm{~min}$.

\section{Results and error analysis}



Fig.4 Time diagram of each bullet train going in and out of process $a, b$ and $c$ AJBAS: https://escipub.com/american-journal-of-basic-and-applied-sciences/ 
Observing the time diagram of each bullet train going in and out of working procedures a, b, c, it is found that:

(1) By comparing the progress time and the time when entering process $a$, it is found that there is a jam when the 9th and 10th motor cars enter process a, that is, there is no spare workshop when the two motor cars enter process $A$. it is calculated that the blocking time of the two cars is 1 minute and 4 minutes respectively.

(2) The first train to leave the whole maintenance system is 286 minutes, and the latest train to leave the whole system is 557 minutes, and the train that leaves the whole system last is not the last train to arrive $a$.

\subsection{Analysis of maintenance sequence}

According to the maintenance results, the maintenance sequence table is listed as follows:

Tab.6 Maintenance sequence list

\begin{tabular}{c|c}
\hline \hline Working procedure & Maintenance sequence \\
\hline Process a & a1-a2-a1-a2-a3-a1-a2-a3-a1-a2-a1 \\
\hline Process b & b1-b2-b3-b4-b5-b1-b2-b3-b4-5b-b6 \\
\hline Process c & c1-c2-c3-c1-c2-c1-c2-c1-c3-c3-c2 \\
\hline \hline
\end{tabular}

According to the observation of the above table, it is found that the workshops in process $b$ have not been fully used, and only 6 workshops have been used at most, and because the arrival time of the train is different, and the process time of different trains is different, the workshops in the process are not used smoothly, so for the workshops in process a, the order is a1-a2-a1a2-a3-a1-a2-a3-a1-a2-a1.

As the process time of each train is different, the proportion of the time of process $a, b$ and $c$ is drawn according to the total time of the four bullet train processes a, b and c, as shown below:

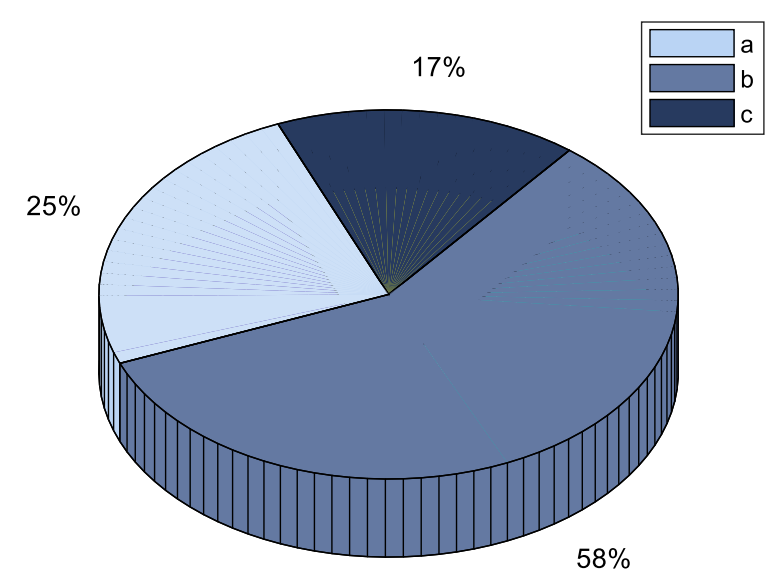

Fig.5 The proportion chart of the duration of processes a, b, c

From the diagram, it is found that process $\mathrm{b}$ accounts for the most time, accounting for $58 \%$ of the total process time, while process $\mathrm{c}$ accounts for the least, accounting for $17 \%$ of the total process time. Through analysis, it is found that the process $a$ in which the workshop vacan- cy occurs. The workshop of the latter process has room and the lack of the workshop of process a will affect the overall maintenance time and maintenance efficiency. Therefore, in connection with practice, the maintenance station can appropriately increase the workshop of process a. 
So as to improve the efficiency of the maintenance chain.

\subsection{Analysis of errors}

Although the problem requires ignoring the transfer time between workshops, it is impossible to maintain the train transfer workshop without time-consuming in real life, and the time spent on workshop transfer will change the queuing rules so as to change the results.

8. Evaluation, improvement and popularization of the model

\subsection{Advantages of the model}

(1) The model is closely related to the actual situation, combined with the actual situation to solve the problems and solve, so that the model is closer to the reality, versatility and generalization. ${ }^{[5]}$

(2) genetic algorithm is used in this paper. Genetic algorithm does not have too many mathematical requirements for the optimization problem solved, and it has good global search ability, it can search areas that have nothing to do with the problem, and multiple individuals can be compared at the same time. And it has strong expansibility, and it is easy to combine with other algorithms. And by making use of its inherent parallelism, it is very convenient to carry out distributed computing and accelerate the speed of solution.

\subsection{Model Disadvantages}

(1) The programming of genetic algorithm is more complex, a large number of functions will be applied in the programming process, and the amount of calculation of genetic algorithm is very large, so the search speed of the algorithm is relatively slow. and the algorithm needs more training time to get a more accurate solution.

(2) Genetic algorithm is difficult to deal with nonlinear constraints, requires multiple operations, and the reliability of the results is poor, cannot get a stable solution, at the same time, it is difficult to deal with or optimize the problem with relatively high dimension.

\subsection{Model improvements}

This paper applies the genetic algorithm, although the genetic algorithm is relatively superior, but there are still some problems in the genetic algorithm, so this paper can consider to further optimize the genetic algorithm and sort all the individuals in the population once. These individuals are listed in descending order according to their fitness, so as to improve the accuracy of the solution and reduce the running time of the algorithm.

\subsection{Generalization of the model}

Genetic algorithm (GA) is an optimal algorithm of global optimization probability, which does not depend on the specific field of the problem and has strong robustness to the types of problems, so it is widely used in many sciences and is suitable for function optimization, combinatorial optimization and job shop scheduling.

In addition, genetic algorithm has also been widely used in production scheduling problems, robotics, image processing, automatic control, genetic coding, artificial life and machine learning.

\section{Conclusion}

With the development of urban modernization and the further development of high-speed rail emus and intelligent detection technology, the application and maintenance industry of EMU develops, and the requirement of train maintenance of high-speed EMU in our country is higher. There are some problems in the process of maintenance, such as high standby rate of EMU, long maintenance and shutdown time, insufficient maintenance capacity and uneven configuration ${ }^{[6]}$. In this paper, the maintenance problem of EMU is discussed, and a mixed nonlinear programming model is established. The genetic algorithm is used to solve the problem, 
and it is found that the shortest total time of all EMU maintenance is $541 \mathrm{~min}$. Through the model established in this paper, the design of the shortest maintenance time in the process of EMU maintenance is realized more forcefully and theoretically, which provides a reference for the optimization of EMU maintenance in actual production and life, and is helpful to improve the maintenance efficiency of EMU.

\section{References}

1. Jinzong Chen, Xin He, Ying Wang, Haiying Li, Jilei Qian. Study on Optimization of maintenance Resource layout of EMU in High Speed Railway [J/OL]. Railway Transportation and economy, 2020 (01): 66mur71 [2020mur02].Https://doi.org/ 10.16668/j.cnki.issn.1003-1421.2020.01.12.

2. Dong Zhao. Model parameter selection based on genetic algorithm and its application in text classification [D]. Hebei University, 2019.

3. Jun Xie, Qian Wang, Yajing $\mathrm{Ni}$, Yingfei $\mathrm{Hu}$, Shuxian Bao. Research status of structural optimization based on genetic algorithm [J]. Journal of Hebei Institute of Architecture and Engineering, 2018,36(03):14-21.

4. Jun Ma. Analysis of matlab solution of genetic algorithm TSP [J]. Science and Technology Horizon, 2018(16):37-38.

5. Qiyuan Jiang. Mathematical modeling [M]. 3rd edition. Beijing: higher Education Press, 2003.

6. Cuijiang Sang. Study on the design standard of maintenance facilities for railway EMU [J]. Technology and economy of Railway Engineering, 2019,34(06):13-18. 